\title{
Simulation of the Conveying Speed on Vibration Conveyors using 1D-Mechanical Modules in SimulationX
}

\author{
Christian Kuhn*, Thomas Risch, Markus Golder \\ Professorship of Conveying Engineering and Materials Handling, Chemnitz University of Technology, Germany \\ * Correspondence: christian.kuhn@mb.tu-chemnitz.de
}

Received 3 November 2020; Accepted 9 November 2020; Available online 7 December 2020

(C) 2020 by C. Kuhn et al. This is an open access article distributed under the Creative Commons Attribution License (CC-BY 4.0), which permits unrestricted use, distribution, and reproduction in any medium, provided the original work is properly cited.

\begin{abstract}
Vibration conveyors are technical systems which generate a stream of materials on basis of vibrations. The vibrations lead to continuous change between acceleration forces and friction. In the practical operation high conveying speeds shall be accomplished with a defined use of the driving force. In this article a simulation model in the software SimulationX is introduced which utilities basic mechanical correlations to calculate the conveying speed. With the help of the presented model influences of vibration movement curve on the conveying speed can be portrayed and analysed.
\end{abstract}

KEYWORDS vibration conveyor, conveying speed, simulation

\section{Introduction}

Vibration conveyors have a long history of use and are regarded as sturdy, hardwearing and reliable conveyor systems. They are utilized in the transport of separate goods as well as bulk goods in all thinkable areas of application. The sizes of vibration conveyors are depending on the application and vary in their length form a few centimetres (e.g. to transport micro components) up to 50 metres (e.g. in mining).

In terms of the logistical planning of vibration conveyors the flow rate of material and the achievable conveying speed are central parameters to focus on during dimensioning. Therefore, it is necessary to determine the suitable parameters of movement during the development process.

In this article the software SimulationX is used to execute the simulation tasks. The software offers convenient possibilities to describe the behaviour of different physical systems.

Interactions between modules can be defined and units of modules can be created by linking individual elements. This way the user has a clear model structure which allows to increase the model depth step by step. 
In the vibration conveying process permanent changes in the state of the conveyed goods between throwing, sliding and sticking result in only partly expedient analytical solutions. Here numerical models can be formulated in a more generalized way.

A comparison with existing calculation models is also discussed in this article.

\section{Simulation Model Design}

The model developed in the context of this article to ascertain the conveying speed rests upon basic mechanical relations between goods to be conveyed and the conveyor organ. This basic model can be set up as shown in Figure 1.

The three basic and essential mechanical components which enable the conveying process as well as the associated coordinate axes and the gravitational acceleration can be identified in Figure 1. At the bottom side the conveyor organ the elastic support is situated. It allows oscillation movements. On the top the goods to be conveyed are carried by the conveyor organ.

During the operation of the conveyor an interaction with the goods to be conveyed takes place through the movement of conveyor organ along the $\mathrm{x}$ and $\mathrm{y}$ axes. As a result of this interaction the goods are set in motion.

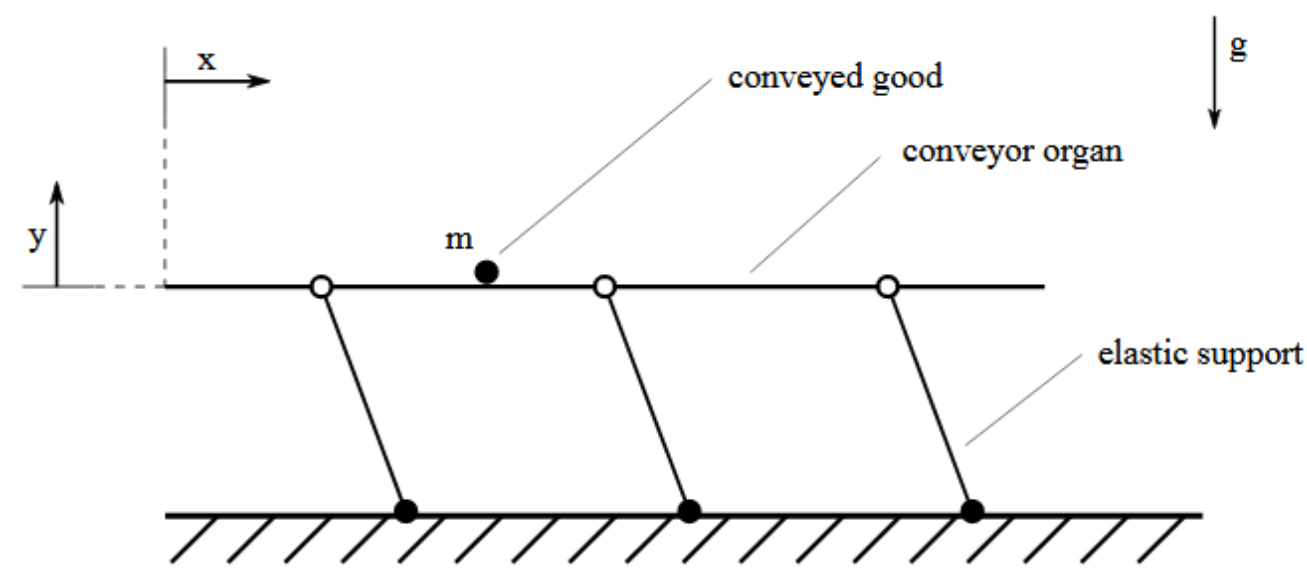

Figure 1: Sketch of a conveyor organ with a piece of good

\subsection{Movement Illustration of the Conveyor Organ}

Matching the mechanical system presented in Figure 1 the equations of motion of the conveyor organ for the two axes of oscillation can be established for a linear conveying process:

$$
x(t)=\cos (\beta) \cdot \frac{a_{A n} \cdot g}{4 \cdot \pi^{2} \cdot f^{2}} \cdot \sin (2 \pi f \cdot t)
$$

and

$$
y(t)=\sin (\beta) \cdot \frac{a_{A n} \cdot g}{4 \cdot \pi^{2} \cdot f^{2}} \cdot \sin (2 \pi f \cdot t)
$$


The equations (1) and (2) are the first input data of the calculation model. The equations determine the position of the conveyor organ as a function of the operation frequency $f$, the throwing angle $\beta$ and the drive acceleration $a_{A n}$ expressed as a factor of the gravitational acceleration. The formulas can be derived by rearranging and inserting them into the context of the standard VDI 2333 [1]. The speed and the acceleration behaviour of the conveyor organ are defined by forming the derivatives of these equations.

At this point alternative and more complicated forms of movement can be integrated into the calculation model by corresponding equations of motion. Here, Risch [2] gives a universal method for any form of movement (Lissajous figures).

\subsection{Force Transfer to the Conveyed Good}

During operating the conveyor organ moves continuously along the given path of movement and influences the goods to be conveyed lying on it. The interaction between the conveyor organ and the conveyed goods on its top occurs through friction between these two elements. According to the assumed friction model, the acceleration forces of the conveyor organ are transferred to the goods. In principle, any friction models can be used to adjust the model depth. However, for most of the practical uses' simple friction models like the Coulomb model meet the requirements. There it is sufficient to determine the friction factor $\mu$ and the normal force.

$$
F_{R}=\mu \cdot F_{N}
$$

with

$$
F_{N}=\left(g+a_{y}\right) \cdot m_{F G}
$$

In the calculation only the friction forces along the $\mathrm{x}$-axis have to be taken into account. In the direction of the $y$-axis the conveyor organ only functions as a mechanical stop level. In case of an upwards movement of the conveyor organ, the mechanical stop moves the goods upwards as well. During the subsequent downward movement, only the gravitational acceleration determines the movement of the goods to be conveyed.

If the gravitational acceleration is less than the backwards acceleration of the conveyor organ along the y-axis the contact state will dissolved and the goods will be thrown. In the state of throwing there are be no further interactions between the goods to be conveyed and the conveyor organ until the goods impacts on conveyor organ again. As soon as the ycoordinates of the goods to be conveyed and the conveyor organ align, the throwing phase ends and the friction contact is re-established. If the level of the backwards acceleration of the conveyor organ remains below the acceleration level of gravity, no goods will be thrown. In this case the friction contact is maintained for the whole movement cycle.

Note: When calculating goods with increased air resistance and vibration parameters with high throwing heights the model should be deepened. The assumed laws of motion in the throwing phase influence the moment of impact. So, they also significantly change the state transition for the subsequent friction contact. In this setting calculated conveyor speeds can differ considerably. This article refrains from further information on this matter. 


\subsection{Setting Up the Calculation Model}

Now a calculation model can be created to form the in the theoretical correlations in the software SimulationX. A possible concept of the model is presented in the Figure 2.

In the realized model the two axes of the linear conveying process are structured as separate event chains. However, the existing interactions between the movement along $\mathrm{x}$-axis and the $y$-axis will not be neglected. By using parameters within the shown element objects the simulation regards the relation between the individual event chains.

The simulation model matches the previous description on how the system functions. At the beginning the movement of the conveyor organ is defined. Accordingly, the position of the conveyor organ is determined over time. In the upper event chain of the $\mathrm{x}$-axis a module to regard friction follows the specification of the conveyor position. If the stop in the second event chain of the $y$-axis is active a friction force is applied in the friction point. At this time the stop delivers the numerical value 1 . With the help of the friction force the goods to be conveyed that are linked as a new module to the friction module are accelerated in the direction of the $\mathrm{x}$-axis.

In the event chain of the $y$-axis there is a mechanical stop instead of the module regarding the friction. The stop describes the degree of freedom of the conveyed goods along the direction of the y-axis. Accordingly, the goods to be conveyed cannot move any lower than the position of the conveyor organ.

Figure 3 illustrates the movement sequences along the direction of the $y$-axis. The curves represent an exemplary conveying process at a frequency of $25 \mathrm{~Hz}$ with an acceleration of $4.3 \mathrm{~g}$ under a throwing angle of $25^{\circ}$. The blue curve shows the continuous movement of the conveyor organ which is defined by the input of equation (2).

The status of contact between the goods to be conveyed and the conveyor organ is displayed in yellow. If the goods to be conveyed are lying on the conveyor organ there is a contact and the curve is at the value 1 . During the contact time the conveyor organ and the goods to be conveyed that are shown in red cover the same distance. With a sufficient acceleration in the $\mathrm{y}$-direction, as in the given example, a throwing phase is initiated as part of the movement sequence. During this phase there is no contact between the goods to be conveyed and the conveyor organ. For this reason, the stop status changes to the value 0 . During the throw the goods to be conveyed also move on a different path above the conveyor organ.

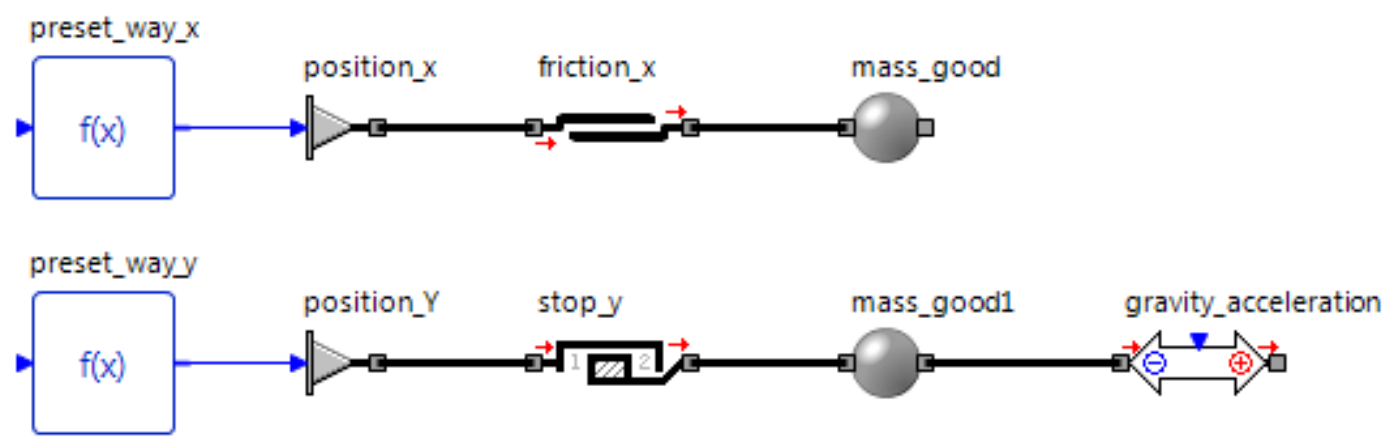

Figure 2: Structure of the event chains of the simulation model 


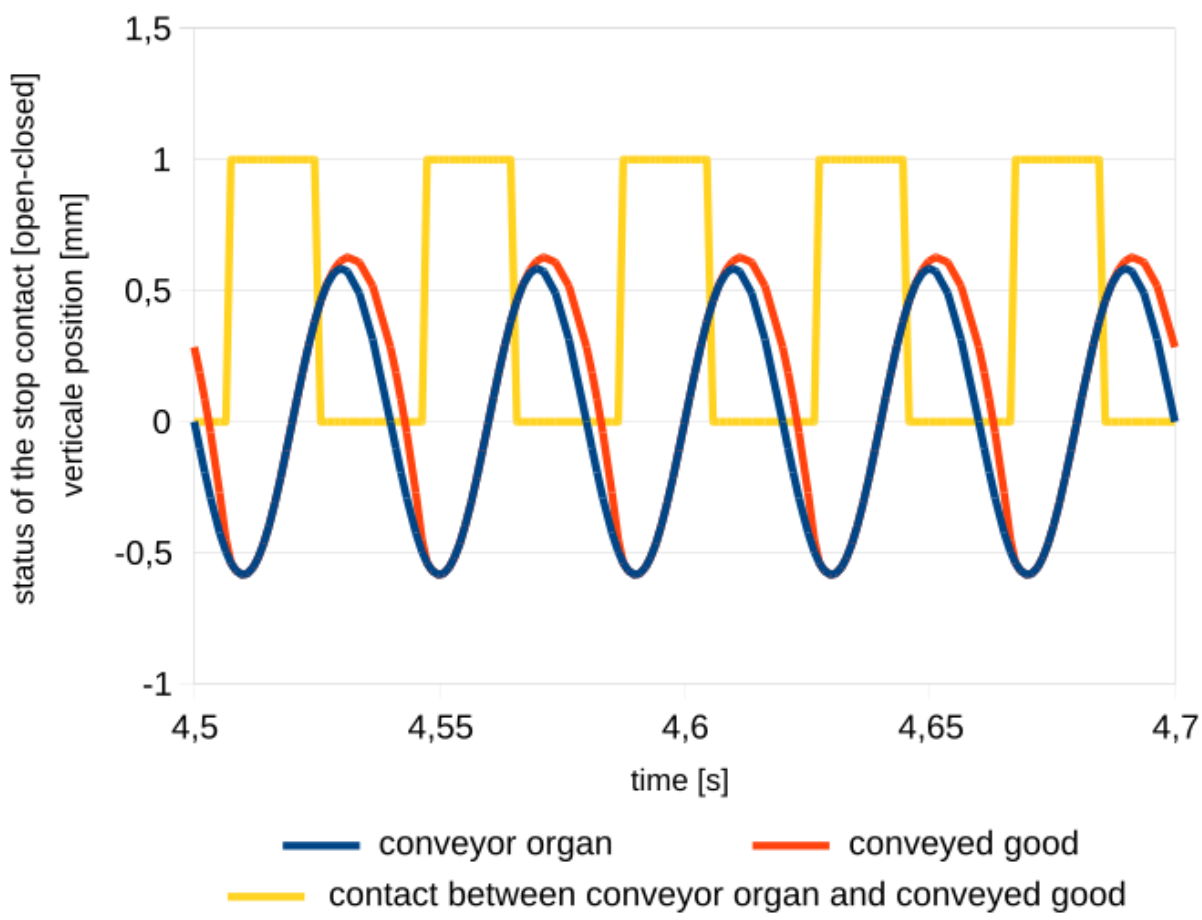

Figure 3: Movement of the conveyor organ and the conveyed goods along the y-axis as well as the status of stop module

In the final step the goods to be conveyed also have to be taken into account in the event chain of the y-axis by a mass element. It is precisely for this mass element that status of the stop is evaluated in the calculation. At this point it is also important to integrate the gravitational acceleration into the calculation model. It influences the goods to be conveyed along the direction of the $y$-axis.

\subsection{Analysis of the Conveying Speed}

The conveying speed of interest for the user is simulated in the event chain of the $\mathrm{x}$-axis. In reality this speed is perceived as a material flow.

However, the conveying speed is not a constant value. It changes depending on the current movement phase of the vibration cycle. That is the reason why for comparison purposes usually the average conveying speed is used. This value can be determined by a simple average value calculation according the equation (5). The average value can be calculated by an integral over an oscillation period. Here it is important that the oscillation has already reached the steady state. Accordingly, the calculation is only to be carried out after reaching the time $t_{s}$ of the steady state.

$$
v_{m}=f \cdot \int_{t_{s}}^{t_{s}+\frac{1}{f}} v(t) \cdot d t
$$

Figure 4 shows the progression of the conveying speeds for the exemplary conveying state at $25 \mathrm{~Hz}$ with an acceleration of $4.3 \mathrm{~g}$ under a throwing angle of $25^{\circ}$. Especially in the speed curve before calculating the average value the phases of the conveying process can be 


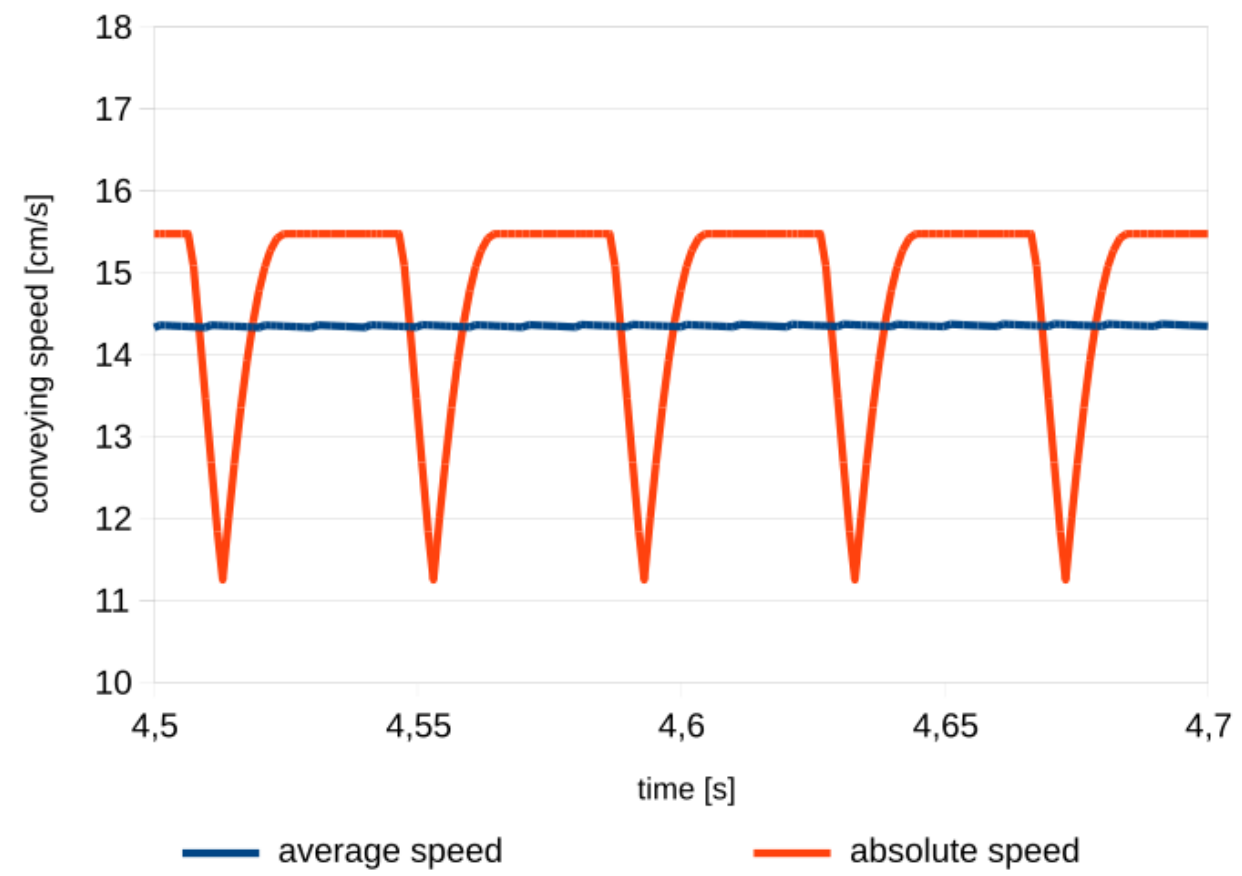

Figure 4: Calculated conveying speed of the given exemplary conveying process

recognised very well. Beginning at the lowest point of the red curve, the goods to be conveyed are accelerated by the friction contact with conveyor organ. The acceleration ends with the goods being thrown whereupon the horizontal part of the curve follows. During the flight phase the good to be conveyed are not influenced by air resistance. This is the reason for the constant speed until the impact of the goods on the conveyor organ. After the impact the conveyed goods are decelerated to the speed of the conveyor organ before the acceleration starts again.

\section{Reference to Existing Calculation Approaches}

The simulation model presented in this article is based on the fundamental mechanical relations between the goods and the conveyor organ that are valid for most of the practical applications of vibration conveyors. Special conditions such as increased air resistance or strong impact behaviour between the goods and the conveyor organ are not taken into account. However, the model structure allows to include such effects to accordingly adjust the model depth at any time. The model relates exclusively to the reaction of the conveyed goods as a result of the conveyor organ movement. Mechanical repercussions on the conveyor organ are not taken into account.

A very detailed view on the mechanical interactions of the conveyed goods can be achieved with a DEM simulation as described by Dallinger in [3]. In contrast to the SimulationX model presented in this article the DEM calculations require very long computing times and more input parameters. For this reason, the SimulationX model described in this article is more suitable for the calculation of conveying processes with low load and to have a first estimation. 
The same mechanical relations as in the presented model are used by Risch in [2]. Therefore, it is understandable that the models also provide comparable results.

In addition, Risch collected the numerical calculation results for a wide range of practically relevant operation parameters. From the collected parameters Risch interpolated analytical approximation functions. These approximation functions are a cause of the deviations from the numerical model in SimulationX presented here.

For the following comparison of the conveying speed between the individual models in Figure 5 the same exemplary conveying process as in section 2.3 was used.

In the curve comparison with the calculation approach according to the VDI standard [1] the area of low throwing angles is particularly noticeable. The curve according the VDI standard in the example in figure 5 only determines a conveying speed different than 0 for larger throwing angles. In this area the model according Risch [2] and the presented SimulationX model calculate slow conveying speeds. The cause is to be found in the consideration of the conveying effect of sliding. The calculation according to the VDI standard neglects the sliding effects and accordingly does not provide any conveying speeds.

In the transition area between sliding and throwing conveying it can be detected that the SimlationX model calculates a significantly sharper transition between the conveying states. The comparatively smoother transition in the model according to Risch can be attributed to the approximation functions used.

After the start of the conveyance by throwing at an angle of about $13^{\circ}$ the three calculated curves approach each other. In this state the SimulationX model shows a course between the two compared models. At higher throwing angles the SimulationX model approaches the results according to the VDI standard.

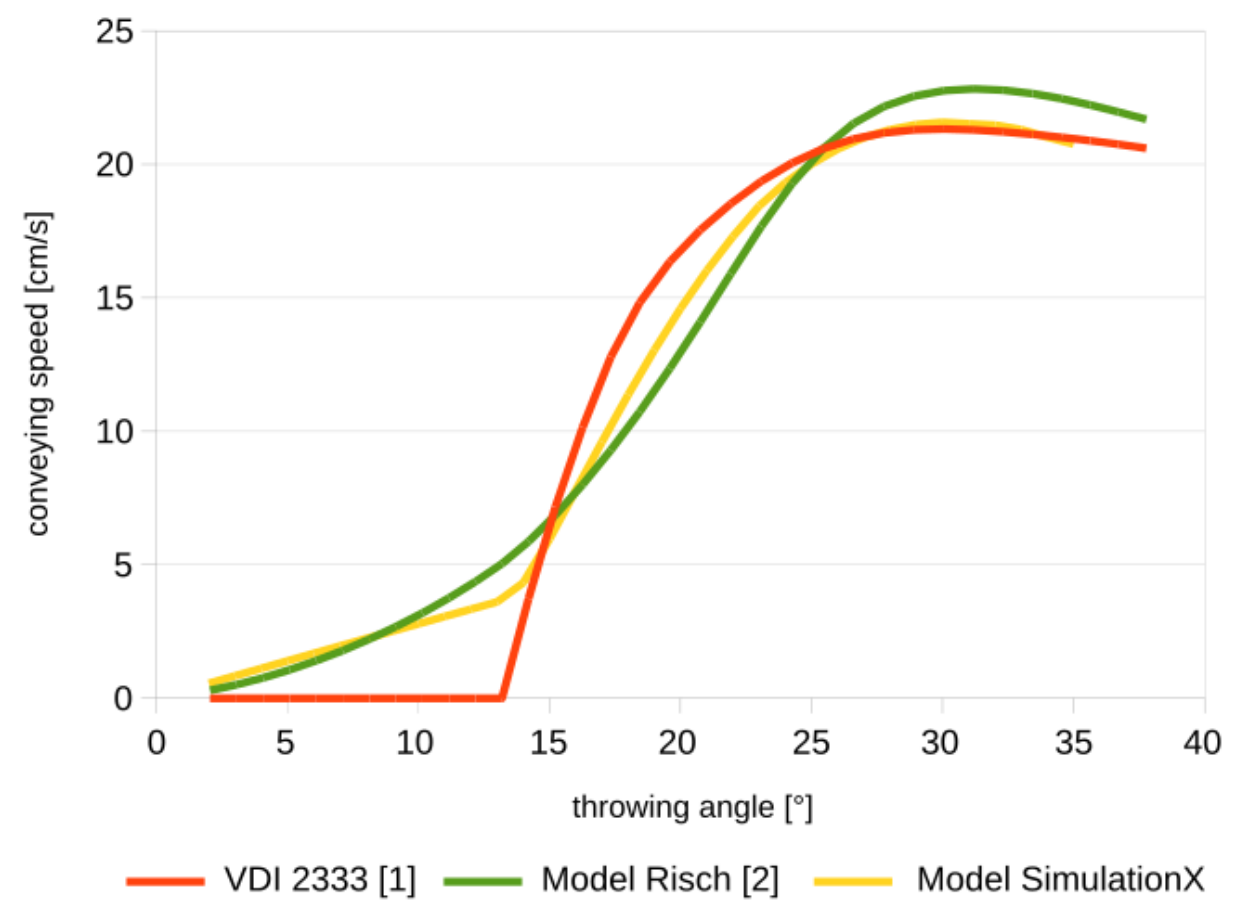

Figure 5: Comparison of the conveying speed between 3 different approaches 


\section{Evaluation of the Model}

The chosen simple simulation setup enables quick calculations and model analysis. At the same time, however, restrictions result from this structure.

At the beginning, when working with the calculation model, the form of movement of the conveying organ has to be known and described as mathematical function. Various assumptions are made when describing the conveyor movement using the equations of motion for the $\mathrm{x}$-axis and $\mathrm{y}$-axis. Thus, for the validity of the calculation, the requirement for the conveyor organ is derived that all points of the conveyor organ behave according to the movement curve. Deviations of the movement due to constructional conditions and the dynamic behaviour of a real conveyor cannot be taken into account (e.g. structural vibrations or tilting effects). Furthermore, the conveyed good is idealized as point mass. This way, specific effects of bulk or piece goods are neglected (cf. [3]).

\section{Conclusion and Outlook}

The model enables the simulation of linear conveyor movements by simple calculations of the conveying speed depending on the movement curve of the conveyor organ. In the calculation, the components of this linear movement are considered individually for each axis and are only linked based on the contact status of the goods to be conveyed.

In the development process of vibration conveyors, the movement curve is not exactly known. By integrating the presented calculation model into a simulation model to calculate the movement curve of the conveyor organ, such as in [4] by Kuhn, the scope of the model can be increased.

The current model structure maps only linear conveyor movements. To increase the versatility, an extension to include non-linear movements is useful. An application case are spiral vibration conveyors, which move the goods on a spiral track. Especially, the widespread use of this design makes a model extension worthwhile.

\section{References}

[1] VDI 2333 Schwingförderer für Schüttgut, Verein Deutscher Ingenieure e.V., Beuth-Verlag, (Berlin, 2017).

[2] Risch, T.: Zweidimensionale Bewegungsformen in der Vibrationsfördertechnik. Dissertation, Chemnitz University of Technology, 2011. https://nbn-resolving.org/urn:nbn:de:bsz:ch1-qucosa-65499

[3] Dallinger, N.: Die Diskrete Elemente Methode als Simulationsmethode in der Vibrationsfördertechnik. Dissertation, Chemnitz University of Technology, 2017. https://nbn-resolving.org/urn:nbn:de:bsz:ch1-qucosa-229364

[4] Kuhn, C., et al.: Simulation of Oscillating Systems for the Usage on Vibration Conveyors with SimulationX. Logistics Journal: Proceedings, 2019, 227-232 (Rostock, 2019). https://doi.org/10.2195/lj_Proc_kuhn_de_201912_01 